and the underlying myelin was also involved. The cause of Generalized Cortical Dysplasia is unknown but a genetic etiologic factor is suspected.

\title{
HEAD SIZE AND COGNITION IN VERY LOW BIRTH WEIGHT (VLBW) INFANTS
}

The effect of VLBW $(<1.5 \mathrm{~kg})$ and subnormal head size at 8 months of age (corrected for prematurity) on neurocognitive abilities at school age was investigated by a longitudinal follow up of 249 children born between 1977 to 1979 and admitted to the neonatal intensive care unit at Rainbow Babies and Children's Hospital, Cleveland, $\mathrm{OH}$. Head size was subnormal at birth in $12 \%$, at term $23 \%$, and at 8 months $13 \%$. As compared with 216 children with normal head sizes, the 33 children with subnormal head sizes at the age of 8 months had significantly lower mean birth weights and higher neonatal risk scores and at 8 years of age had a higher incidence of neurologic impairment and lower IQ scores. A subnormal head size at 8 months of age was predictive of poor verbal and performance IQ scores at 8 years of age; lower scores for receptive language, speech, reading, mathematics and spelling; and a higher incidence of hyperactivity. (Hack M et al. Effect of very low birth weight and subnormal head size on cognitive abilities at school age. N Engl J Med July 25, 1991; $\underline{325: 231-237)}$

COMMENT. A relation between subnormal head circumference and cognitive functioning has been reported in many different studies, including children with learning disabilities. Improvements in neonatal growth and development may depend on nutrition of chronically ill neonates and environmental enrichment after discharge from hospital. This study shows that the determination of the head circumference at 8 months of age is a useful means of predicting subsequent cognitive deficits and difficulties in school. Volpe JJ, in an editorial (N Engl J Med 1991; 325:276-277), stresses the need for a better method of detection of brain injury in VLBW infants since $77 \%$ of those with normal head circumference at 8 months had IQs below 85 and an academic skill index below 80 in the study by Hack et al. Those infants with a small head circumference at 8 months of age made up only a minority of infants who subsequently had cognitive deficits. Cranial ultrasonography detects overt brain lesions but not the subtle lesions in the periventricular white matter. MRI may detect periventricular injury missed by ultrasound and CT scanning; it is also used to measure the progress and degree of myelination in the human infant in the first months of life. A decreased rate of brain growth in the first months of life might identify a larger proportion of at risk infants and justify further study with the MRI in selected patients.

Collin MF and associates from the Department of Pediatrics, Loyola University Medical Center, Maywood, IL report that normal infant development is poorly predictive of continued normal development in the extremely low birth weight infant (Pediatrics July 
1991 ; $\underline{88}: 115-120)$. Only 11 (31\%) of 36 children had normal development upon reassessment in early childhood. With or without major complications, extremely low birth weight places children at risk for emerging development problems with age. Head circumference was not addressed in this study.

\section{PRENATAL ETIOLOGY OF CEREBRAL PALSY}

The incidence of minor malformations in a group of 137 adults with cerebral palsy, living in a New York State residential institution, is reported from the Division of Developmental Pediatrics, Robert Warner Rehabilitation Center, State University of New York, Buffalo, NY. Subjects were classified in three etiological groups: (1) prenatal onset, (2) postnatal onset, and (3) perinatal onset. Criteria for enrollment in group 1 included a known prenatal cause (i.e., CNS malformation, chromosomal aberration or congenital infection) or fullterm birth with unremarkable delivery and neonatal course. Of 109 patients with CP of prenatal origin, $82 \%$ had unknown causes, $10 \%$ CNS malformation, $5 \%$ chromosomal aberration and $3 \%$ congenital infection. Of 28 patients with postnatal causes, $39 \%$ had bacterial meningitis, $32 \%$ encephalitis, $11 \%$ metabolic disease, $7 \%$ cardiopulmonary arrest and $7 \%$ trauma. Spastic quadriplegia, profound mental retardation, and seizures occurred in $80-90 \%$ of the total sample, and the neurological findings were similar in the two groups. The prenatal group with known etiology and a subgroup with unknown etiology had significantly more minor malformations than the postnatal group. Individuals with $\mathrm{CP}$ and multiple minor malformations, in the absence of definitive postnatal or perinatal factors, have CNS developmental dysfunction caused most likely by prenatal influences. (Coorssen EA et al. Multiple minor malformations as a marker for prenatal etiology of cerebral palsy. Dev Med Child Neurol August 1991; $\underline{33}: 730-736$ ).

COMMENT. Increased numbers of minor malformations are indicative of aberrant embryonic development of the CNS (Illingworth R. "Why blame the obstetrician? A review." BMJ 1979; 1:797-801) Less than $10 \%$ of $\mathrm{CP}$ is caused by perinatal asphyxia or events related to delivery (Blair E, Stanley F. "Intrapartum asphyxia: a rare cause of cerebral palsy." J Pediatr 1988; 112:515-519).

\section{INFANTILE SEIZURES}

\section{INFANTILE SPASMS AND PARTIAL SEIZURES}

The concurrence of infantile spasms and partial seizures was studied in 11 infants using time-locked video electroencephalography at the Department of Pediatrics, Children's Hospital, Ohio State University School of Medicine, Columbus, OH. Partial seizures preceded the onset of infantile spasms in 7 . In some, the focal EEG ictal activity was overriding the generalized slowwave transient and in others was visible only during the generalized decrement. Interictal EEGs showed hypsarrhythmia in 6 patients and modified hypsarrhythmia in 5. Brain malformations occurred in 3 and tuberous 